\title{
Satisfação no trabalho do pessoal de enfermagem na rede pública de saúde em uma capital brasileira
}

\author{
Job satisfaction of nursing staff in the public health network in a \\ Brazilian capital city
}

Ada Ávila Assunção (https://orcid.org/0000-0003-2123-0422) ${ }^{1}$

Adriano Marçal Pimenta (https://orcid.org/0000-0001-7049-7575) ${ }^{2}$

${ }^{1}$ Departamento de Medicina Preventiva e Social. Universidade Federal de Minas Gerais. Av. Alfredo Balena $190,6^{\circ}$ andar, sala 630, Santa Efigênia. 30310150 Belo Horizonte MG Brasil.adavila@medicina. ufmg.br

${ }^{2}$ Departamento de Enfermagem MaternoInfantil e Saúde Pública. Universidade Federal de Minas Gerais. Belo Horizonte MG Brasil.

\begin{abstract}
Evidence confirms that the satisfaction perceived by the nursing staff is associated with indicators of the professionals' well-being and quality of the provided services. The aim of this cross-sectional study was to investigate factors associated with reports of job satisfaction. Job satisfaction was assessed based on the validated question: "Are you satisfied with your work?". Stratified and proportional random sampling included nurses, nursing aides and technicians from the municipal health network. The multivariate model was based on the Poisson regression technique with robust variances to estimate factors independently associated with job satisfaction. A total of 290 subjects participated: $73.8 \%$ of them reported job satisfaction. Age, having strong social support and better working conditions were positively associated with satisfaction. Positive screening for common mental disorders was negatively associated with satisfaction. The emphasis on the work environment and type of management indicates the relevance of these factors for the strengthening of human resources in health.
\end{abstract}

Key words Job satisfaction, Nursing, Nursing team, Working conditions
Resumo Evidências confirmam que a satisfação no trabalho percebida pelo pessoal da enfermagem está associada a indicadores de bem-estar dos profissionais e qualidade dos serviços prestados. $O$ objetivo do estudo transversal foi investigar fatores associados com o relato de satisfação no trabalho. A satisfação no trabalho foi avaliada com base na pergunta já validada: "Você está satisfeito com o seu trabalho?". A amostragem aleatória estratificada e proporcional incluiu enfermeiros, auxiliares e técnicos de enfermagem da rede municipal. O modelo multivariado foi baseado na técnica de regressão de Poisson com variâncias robustas para a estimação dos fatores independentemente associados com a satisfação no trabalho. Participaram 290 sujeitos: 73,8\% relataram satisfação com o trabalho. Idade, contar com forte apoio social e melhores condições de trabalho foram positivamente associadas com satisfação. A triagem positiva para transtornos mentais comuns foi negativamente associada com satisfação. O destaque para o ambiente de trabalho e tipo de gestão indica a relevância desses fatores para o fortalecimento dos recursos humanos em saúde.

Palavras-chave Satisfação no trabalho, Enfermagem, Equipe de Enfermagem, Condições de Trabalho 


\section{Introdução}

O trabalho de enfermagem é reconhecidamente fundamental para a qualidade da prestação dos serviços, além de essencial para garantir a produtividade das organizações de saúde ${ }^{1,2}$. A jornada desses profissionais é marcada por situações tensas, haja vista o contexto do atendimento que reflete em algum grau as mudanças demográficas e epidemiológicas que ocorrem em uma conjuntura de crise de financiamento no setor ${ }^{3}$. Essas mudanças recentes demandam a construção de habilidades e procedimentos específicos para lidar, por exemplo, com problemas neuropsiquiátricos graves, sintomas relacionados ao uso de substâncias, sofrimento ocasionado por abuso e violência, entre outras situações que trazem os cidadãos aos serviços. Surtos epidêmicos, catástrofes humanas e ambientais incrementam exponencialmente o volume de demandas por atendimento ${ }^{4-6}$. É possível que essas circunstâncias estejam sobrecarregando o pessoal da enfermagem $^{7-9}$, porque nem sempre os serviços estão amparados pela adequação da infraestrutura e ambiente laboral ao desenvolvimento da ativida$\mathrm{de}^{5,6}$. No conjunto são fatores relacionados à percepção de satisfação no trabalho ${ }^{1,5,10}$. Por sua vez esse construto está associado tanto com a saúde desses profissionais ${ }^{10,11}$ quanto com a qualidade dos serviços prestados ${ }^{12}$ e intenção de mudar de emprego ou de abandonar a profissão ${ }^{13-15}$.

Motivação é um conceito chave para abordar a adesão dos trabalhadores aos sistemas e seus objetivos, porque é adjacente à noção de engajamento no projeto organizacional. A motivação está relacionada à satisfação dos trabalhadores, que é um construto multidimensional, cuja expressão seria resultado de uma comparação entre aquilo que foi obtido e aquilo que se desejou obter do trabalho ${ }^{12}$. Sob esse prisma, trata-se de um sentimento sobre o trabalho, que reflete, como esclareceu Spector ${ }^{16}$, em que medida as pessoas gostam ou não gostam do seu trabalho. Diz respeito, portanto, a uma constelação de atitudes relacionadas a vários aspectos do ambiente labo$\mathrm{ral}^{12}$.

Em breve, o julgamento dos resultados da atividade de trabalho está na base da satisfação percebida pelos enfermeiros, auxiliares e técnicos de enfermagem ${ }^{1,17}$. Certo, a percepção de satisfação é subjetiva, além de variar de acordo com as características pessoais, amadurecimento e realização em outros âmbitos da vida ${ }^{18,19}$. Essa constatação, entretanto, não autoriza desconsiderar a satisfação no trabalho, haja vista a associação confirma- da com a qualidade dos serviços prestados ${ }^{5,13,18}$. Isso porque o grau de satisfação laboral tem a ver com aquilo que os profissionais identificam como fatores facilitadores ou constrangedores de suas práticas ${ }^{12,20}$. Embora esteja reconhecida melhor qualidade dos serviços prestados quando os profissionais se sentem satisfeitos no trabalho ${ }^{5}$, nessa esfera faltam evidências no Brasil. Atualizações no tema são necessárias, tanto no que se refere a produção de dados empíricos, quanto às elaborações que permitam maior compreensão dos mecanismos subjacentes às associações encontradas, a fim de ampliar e aprofundar o conhecimento e indicar pistas para a elaboração das políticas específicas no campo dos recursos humanos em saúde.

O objetivo foi identificar fatores associados ao relato de satisfação no trabalho em uma amostra de auxiliares e enfermeiros dos serviços do sistema público municipal de saúde de uma capital brasileira.

\section{Métodos}

Trata-se de um estudo transversal, conduzido, entre 2008 e 2009, com trabalhadores de enfermagem em efetivo exercício profissional na rede municipal de saúde.

À época da coleta de dados, foram considerados elegíveis todos os profissionais de enfermagem que trabalhavam na assistência direta ao paciente (níveis superior e médio), independentemente do vínculo empregatício. A amostra foi dimensionada em 285 sujeitos, com base nos seguintes parâmetros: $70 \%$ de satisfação com o trabalho em enfermagem ${ }^{18}$, nível de confiança de 95\%, erro de 5\%. Ao final, foram incluídos 297 participantes no estudo. Houve 7 perdas devido a incompletude da resposta fundamental para constituir a variável resposta.

A seleção dos participantes seguiu a amostragem aleatória estratificada e proporcional baseada em área geográfica e nível de complexidade da assistência (centros de saúde, ambulatório de especialidades, unidades de urgência ou na gerência distrital). A distribuição proporcional dos sujeitos de acordo com os dois estratos (auxiliares e enfermeiros) foi baseada na lista de funcionários fornecida pelo departamento de recursos humanos da Prefeitura Municipal. O sorteio se deu por meio de números aleatórios gerados pelo Epi-Info (versão 3.5.1).

Os sujeitos sorteados para participar que não se encontravam no serviço por motivo de férias, 
transferência, aposentadoria ou morte foram substituídos, respeitando-se o território geográfico e o nível de complexidade assistencial.

A coleta de dados foi realizada entre setembro de 2008 e janeiro de 2009, com o auxílio de um questionário autoaplicado, previamente testado em um estudo piloto, contando com perguntas relativas a características sociodemográficas e condições de trabalho. Estas últimas foram organizadas nos seguintes termos: características gerais do trabalho, condições psicossociais (demanda, controle, apoio social); condições ambientais (ventilação, temperatura, iluminação, recursos técnicos e equipamentos do local de trabalho) e carga física de trabalho.

\section{Variável de desfecho}

A satisfação com o trabalho foi mensurada com base na seguinte pergunta: "Você está satisfeito com o seu trabalho?". As seguintes opções de resposta estavam disponíveis: a) "Não estou satisfeito de forma nenhuma", b) "Não estou satisfeito", c) "Estou satisfeito", d) "Estou muito satisfeito". Para a análise dos dados, as opções a) e b) foram agrupadas como categoria "Não" e as opções c) e d) foram agrupadas como categoria "Sim".

\section{Variáveis de exposição}

As variáveis incluídas no estudo foram classificadas em três blocos:

1) Fatores sociodemográficos e de estilo de vida $=$ sexo (masculino, feminino); idade (20-29 anos, 30-39 anos, 40-49 anos, 50 e mais anos; em algumas análises foi usada de maneira contínua); cor da pele (branca, parda/preta, amarela/indígena); estado civil (solteiro, casado/união estável, divorciado/viúvo), renda familiar (até 2 salários mínimos, 2 a 4 salários mínimos, 4 e mais salários mínimos - o salário mínimo à época era de R\$ 415,00); tabagismo [Considerando como fumante quem já fumou pelo menos 100 cigarros, ou 5 maços, você se classifica como? (Não-fumante, Ex-fumante, Fumante atual)]; dependência alcoólica (com base no questionário Cut Down, Annoyed by critcism, Guilty and Eye-opener-CAGE: não, sim) ${ }^{21}$; atividade física [Com que frequência você realiza atividades físicas? (Nunca, 1 a 2 vezes por semana, 3 ou mais vezes por semana)]; atividade de lazer (não, sim).

2) Condições de saúde = falta por motivo de saúde no último ano (não, sim); licença por motivo de saúde no último ano (não, sim); diagnóstico de enfermidade laboral [Você já teve doença de trabalho diagnosticada? (não, sim)]; diagnóstico de doença crônica não transmissível - DCNT [Resposta positiva ao diagnóstico médico de uma ou mais das seguintes enfermidades: diabetes, colesterol alto, obesidade, pressão alta, câncer, cardiopatia (não, sim)]; diagnóstico de doença infecciosa ou parasitária - DIP [Resposta positiva ao diagnóstico médico de uma ou mais das seguintes enfermidades: tuberculose, hepatite, infecção urinária (não, sim)]; diagnóstico de doença do aparelho respiratório - DAR [Resposta positiva ao diagnóstico médico de uma ou mais das seguintes enfermidades: rinite/sinusite, asma, disfonia (não, sim)]; triagem positiva para transtorno mental comum - TMC. Foi aplicado o Self Reporting Questionnaire (SQR-20), um instrumento desenvolvido pela Organização Mundial da Saúde para avaliar morbidade psíquica em países em desenvolvimento ${ }^{22}$. O questionário contém 20 perguntas sobre sintomas emocionais e físicos relacionados a quadros psiquiátricos. Considerou-se a triagem positiva para TMC quando o participante respondeu sim a sete ou mais questões, padrão adotado em outros estu$\operatorname{dos}^{23}$; diagnóstico médico de doenças osteomusculares relacionadas ao trabalho (não, sim); diagnóstico médico de lombalgia (não, sim).

3) $\mathrm{Na}$ dimensão "condições de trabalho" foram abordados categoria profissional (enfermeiro, auxiliar/técnico de enfermagem); tempo no trabalho atual em meses $(<12,12$ a 23,24 a $47, \geq 48$ ); carga horária semanal (até 40 horas, mais de 40 horas); exposição ao trabalho noturno (não, sim); demanda-controle do trabalho (baixa exigência, ativo, passivo e alta exigência); apoio social no trabalho (baixo, alto); condições do ambiente de trabalho (precárias, satisfatórias, ótimas); carga física no trabalho (baixa, média, alta); comprometimento excessivo com o trabalho (baixo, médio e alto); demanda emocional do trabalho [Meu trabalho me exige muito emocionalmente (discordo fortemente e discordo = baixa, concordo e concordo fortemente = concordo)]; exposição a agentes biológicos/químicos [Baseado em quatro perguntas: a) Você entra em contato com materiais biológicos, como sangue, fezes, urina, saliva, líquido amniótico, etc?, b) Você entra em contato com radiação ionizante, como raio $\mathrm{X}$, tomografia, radiografia, radiofármacos?, c) Você entra em contato com gases anestésicos?, d) Você entra em contato com agentes citotóxicos/quimioterápicos?. As quatro perguntas tinham as seguintes opções de resposta: nunca, raramente, às vezes, sempre. Assim, foi criado um escore com o somatório das respostas 
às perguntas que posteriormente foi organizado em tercis (baixa, média, alta)]; comprometimento excessivo com o trabalho (baixo, médio, alto).

Para a caracterização das "condições psicossociais do trabalho" foi utilizado o Job Content Questionnaire (JCQ) proposto por Karasek ${ }^{24}$, validado na versão em português para a população brasileira $^{25}$. A demanda de trabalho foi estimada a partir de um escore resultante do somatório dos valores referentes às questões relacionadas ao ritmo de trabalho, tempo para a realização das tarefas, tarefas conflitantes e volume excessivo de trabalho $^{24,25}$. Para o controle sobre o próprio trabalho, também, foi construído um escore resultante do somatório dos valores referentes às questões relacionadas ao uso de habilidades (aprendizagem de coisas novas, criatividade, desenvolvimento de habilidades especiais e possibilidade de realização de diferentes tarefas) e à autoridade decisória (liberdade para decidir como realizar as tarefas e possibilidade de tomada de decisões). As perguntas eram seguidas das opções de resposta: "concordo fortemente", "concordo", "discordo" e "discordo fortemente", sendo que cada uma delas recebeu uma pontuação de 1 a 4 (1 indicava pouca demanda ou pouco controle e 4 , muita demanda ou muito controle). Os escores de demanda e de controle foram divididos em duas metades com base em suas medianas e, posteriormente, procedeu-se a combinação dessas frações, gerando quatro quadrantes: (a) baixa exigência = baixa demanda e alto controle; (b) ativo = alta demanda e alto controle; (c) passivo = baixa demanda e baixo controle; $(\mathrm{d})$ alta exigência $=$ alta demanda e baixo controle. A alta exigência, proxy do estresse no trabalho, tem se mostrado associado a repercussões negativas sobre a saúde ${ }^{23,24}$. No que diz respeito ao apoio social no trabalho, o JCQ contém perguntas que envolvem o suporte dos colegas de trabalho e da chefia, apresentando como opções de resposta: "concordo fortemente", "concordo", "discordo" e "discordo fortemente", sendo que, cada uma delas recebe uma pontuação de 1 a 4 ( 1 indica pouco suporte e 4 , muito suporte). O escore de apoio social foi dividido em duas metades com base na mediana ${ }^{24,25}$.

A variável "condições ambientais de trabalho" tem sido utilizada ${ }^{23}$ para agregar as respostas a perguntas sobre ventilação, temperatura, iluminação, recursos técnicos e equipamentos. Sobre esses fatores, as respostas possíveis foram: precárias (1), razoáveis (2), ou ótimas (3). Para a variável ruído originado no trabalho e fora dele as opções de resposta foram: insignificante (3), razoável (2), alto e insuportável (1). Ao final, foi realizado o somatório das respostas para cada item a fim de construir um escore global, segundo o qual, quanto maior a pontuação, melhor as condições do ambiente de trabalho.

Para a variável "carga física no trabalho", foi considerado, conforme publicação anterior ${ }^{22}$, as respostas às questões sobre posturas associadas a dor ou desconforto; posição sentada ou em pé por períodos prolongados; transporte manual ou levantamento de peso excessivo ou movimentação do paciente no leito, por exemplo; e, existência de pausas durante o trabalho (11/4 nunca, $2 \frac{1 / 4}{4}$

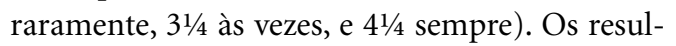
tados foram tratados de maneira a construir um escore global por meio do somatório das respostas para cada item. Para a análise dos dados, o escore foi classificado em tercis (baixa, média, alta).

A variável "comprometimento excessivo com o trabalho" foi construída a partir da subescala de seis questões relativas ao comprometimento com o trabalho do Effort-Reward Imbalance Model (ERI) proposto por Siegrist, sendo adaptado para o português brasileiro ${ }^{26}$. O conteúdo dessas seis perguntas aborda a pressão do tempo para a realização das atividades de trabalho, o despertar pela manhã já com o foco nos problemas do trabalho, a dificuldade de se "desligar" facilmente do trabalho após a jornada laboral, a opinião de pessoas íntimas que consideram que o indivíduo se sacrifica muito pelo seu trabalho, a dificuldade de não conseguir parar de pensar no trabalho quando vai dormir e, a dificuldade de dormir ao adiar alguma tarefa de trabalho. As opções de resposta para as seis perguntas são: discordo fortemente, discordo, concordo e concordo fortemente. Ao final, foi realizado o somatório das respostas para cada item para a construção de um escore global. Para a análise dos dados, esse escore foi organizado em tercis(baixo, médio, alto).

As características da amostra foram apresentadas de acordo com o cálculo das frequências absolutas e relativas das variáveis sociodemográficas, estilo de vida, situação de saúde e condições de trabalho. Adicionalmente, testou-se a associação de cada uma dessas variáveis com a satisfação no trabalho.

As variáveis reconhecidas na literatura como sendo associadas à satisfação no trabalho, assim como, aquelas que apresentavam significância estatística inferior a $20 \%(\mathrm{p}<0,20)$ durante a análise bivariada foram consideradas como candidatas ao modelo final. Este modelo foi construído com técnica de regressão de Poisson com variâncias robustas, e a estratégia passo a passo, no qual se incluiu de todas as variáveis selecionadas 
durante a análise bivariada em ordem decrescente de significância estatística.

Foi realizada, ainda, mais uma estratégia de análise multivariada com a elaboração de um modelo de acordo com o quadro teórico. Em tal modelo, são dispostos três blocos de variáveis já descritos anteriormente [ 1 - demográficas, socioeconômicas e do estilo de vida (distal); 2 - situação de saúde (intermediárias); 3 - condições de trabalho (proximais)]. Dessa feita, a modelagem foi realizada em três etapas. Na primeira, assim como para o modelo passo a passo, as variáveis consolidadas na literatura como fatores associados à satisfação no trabalho, e aquelas que apresentavam significância estatística inferior a $20 \%$ $(\mathrm{p}<0,20)$ durante a análise bivariada, foram selecionadas para a segunda etapa. Nessa segunda etapa, as variáveis com significância estatística inferior a $10 \%(\mathrm{p}<0,10)$ dentro de cada bloco foram selecionadas para a terceira etapa. Na terceira etapa, dentro de cada bloco, as variáveis foram incluídas no modelo final em ordem decrescente de significância estatística, entrando, inicialmente, aquelas do grupo proximal, seguidas das do grupo intermediário e, por fim, as do grupo distal.

Em ambas as técnicas de modelagem estatística, as variáveis que apresentaram $\mathrm{p}<0,05$ foram retiradas uma a uma do modelo e consideradas definitivamente excluídas, se o decréscimo na explicação do desfecho não fosse estatisticamente significativo. Para analisar esse parâmetro, o modelo foi avaliado a cada retirada com o auxílio dos testes estatísticos de Wald e a razão de verossimilhança parcial baseado na estatística $-2[\ln (\operatorname{Lr})-\ln (\mathrm{Lc})]$, que compara a verossimilhança do modelo reduzido - (Lr) com a verossimilhança do modelo completo - (Lc). Todas as análises estatísticas foram conduzidas com o software STATA (versão 12.0).

O estudo foi aprovado por Comitê de Ética e Pesquisa em Seres Humanos. Todos os participantes assinaram o Termo de Consentimento Livre e Esclarecido (TCLE).

\section{Resultados}

Participaram do estudo 290 profissionais, 143 $(49,3 \%)$ eram enfermeiros e $147(50,7 \%)$ eram técnicos ou auxiliares de enfermagem. A maioria dos participantes, 73,8\%, relataram estar satisfeitos com o seu trabalho. As características sociodemográficas e estilos de vida, e as suas associações com a satisfação no trabalho em enfermagem são apresentadas na Tabela 1. Verificou- se que a maioria era do sexo feminino $(89,8 \%)$, tinha entre 30 e 49 anos de idade $(61,4 \%)$, cor de pele parda/preta (61\%), era casada/união estável $(52,1 \%)$ e ganhava até 4 salários mínimos $(65,6 \%)$. Ademais, as seguintes frequências de hábitos de vida estavam presentes entre eles: $11,7 \%$ de tabagismo; 5,5\% de dependência alcoólica, $49 \%$ de sedentarismo e $66,9 \%$ de realização de atividade de lazer. A idade $\geq 50$ anos se associou positivamente com a satisfação com o trabalho $(\mathrm{p}=0,045)$.

A situação de saúde e as suas associações com a satisfação no trabalho em enfermagem são apresentadas na Tabela 2. Observou-se que 58,1\% e $45,2 \%$, respectivamente, faltaram ou se licenciaram do trabalho por motivo de saúde. No que diz respeito ao diagnóstico médico de doenças, os participantes relataram $13,1 \%$ de enfermidade laboral, $41 \%$ de DCNT, $7,2 \%$ de DIP, $35,2 \%$ de DAR, $27,2 \%$ de TMC, $9 \%$ de LER/Dort e 29,7\% de lombalgia. O TMC se associou negativamente com a satisfação com o trabalho $(\mathrm{p}<0,001)$.

As condições de trabalho e as suas associações com a satisfação no trabalho em enfermagem são apresentadas na Tabela 3. Observou-se que a maioria ocupava o cargo de enfermeiro (50,7\%), estava no trabalho atual há mais de 48 meses (59\%), executava carga horária semanal de até 40 horas $(60,3 \%)$, trabalhava no período diurno (75,5\%). Quanto às condições psicossociais de trabalho, a maioria relatava estresse no trabalho de moderada à alta intensidade segundo a escala de demanda-controle (56,6\%: passivo $=33,8 \%$ e alta exigência $=22,8 \%$ ), contava com alto apoio social no trabalho $(50,3 \%)$, e desenvolviam tarefas sob demanda emocional $(84,8 \%)$. As demais variáveis foram classificadas em tercis (condições do ambiente de trabalho, carga física de trabalho, exposição a riscos ambientais (agentes biológicos/químicos) e comprometimento excessivo com o trabalho), ainda que tenha havido alguns desequilíbrios entre as categorias devido às características das suas distribuições.

O alto apoio social, as ótimas condições de trabalho se associaram positivamente com a satisfação com o trabalho $(\mathrm{p}<0,05)$. Por outro lado, a categoria ativo da escala demanda-controle, as categorias regular e ruim/muito ruim da relação entre exigências das tarefas/recursos disponíveis, a alta demanda física e o comprometimento excessivo com o trabalho se associaram negativamente com a satisfação com o trabalho $(\mathrm{p}<0,05)$ (Tabela 3$)$.

Na Tabela 4, é apresentada a análise multivariada para a associação independente entre as 


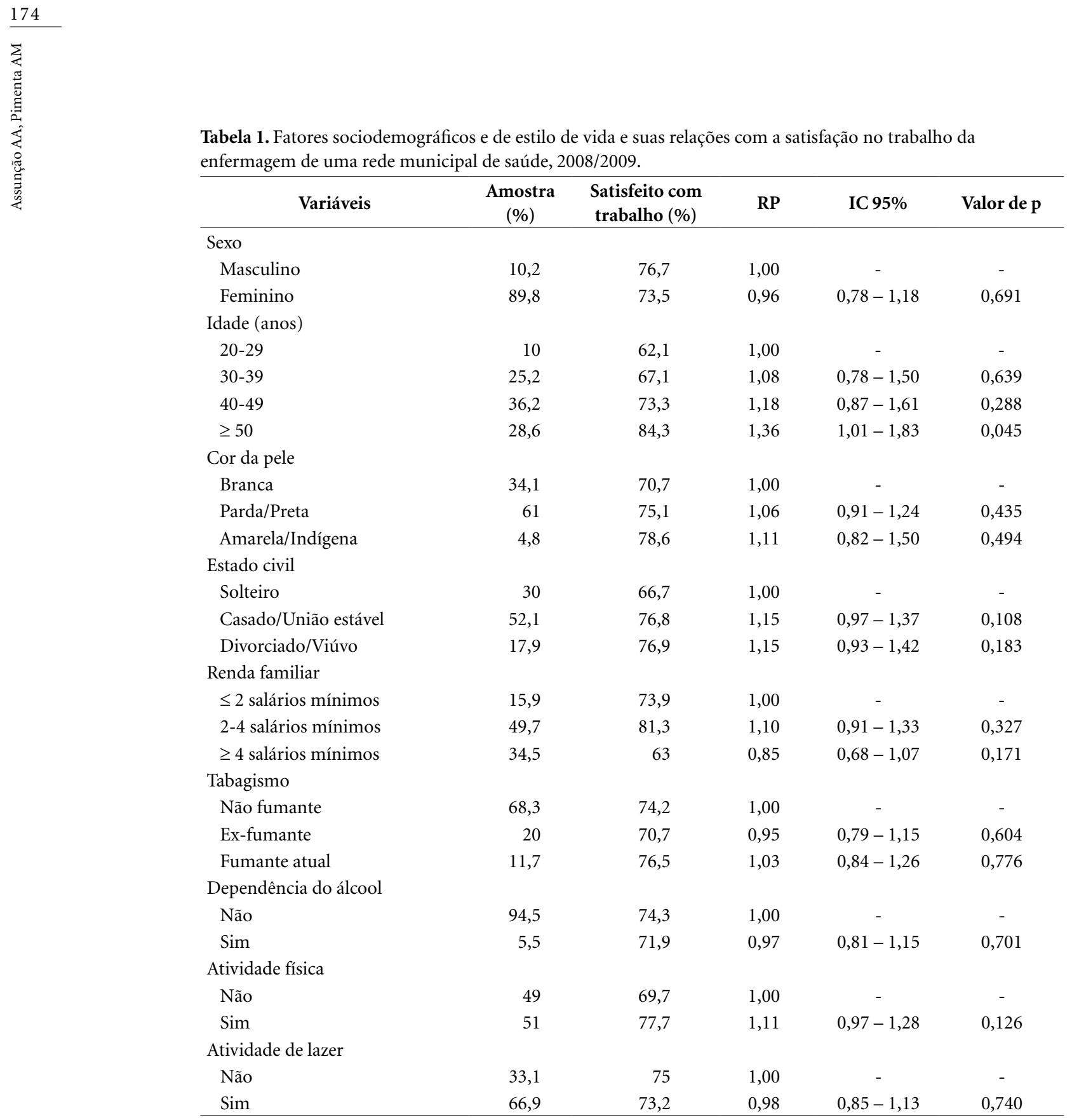

covariáveis e a satisfação com o trabalho. Tanto a técnica passo a passo quanto a em blocos produziu o mesmo modelo final. Nele verifica-se que a idade, o alto apoio social e melhores condições ambientais de trabalho se associaram positivamente com a satisfação com o trabalho. Assim, a cada ano de incremento da idade aumentou-se a satisfação com o trabalho em $1 \%$. Além disso, o alto apoio social, as condições ambientais satisfatórias e ótimas aumentaram a satisfação com o trabalho, respectivamente, em $17 \%, 18 \%$ e $25 \%$. Por fim, ressalta-se que a probabilidade de TMC permaneceu negativamente associado de forma independente à satisfação com o trabalho. Dessa forma, observou-se diminuição em $32 \%$ da satisfação no trabalho nos participantes cujo relato foi compatível com a probabilidade de TMC.

\section{Discussão}

Avançar em idade, perceber-se apoiado no desenvolvimento das tarefas e informar melhores condições ambientais de trabalho foram associados de forma consistente com o relato de satisfação pelos sujeitos da amostra, que incluiu en- 
Tabela 2. Situação de saúde e suas relações com a satisfação no trabalho da enfermagem de uma rede municipal de saúde, 2008/2009.

\begin{tabular}{|c|c|c|c|c|c|}
\hline Variáveis & $\begin{array}{c}\text { Amostra } \\
(\%)\end{array}$ & $\begin{array}{c}\text { Satisfeito com } \\
\text { trabalho }(\%)\end{array}$ & $\mathbf{R P}$ & IC 95\% & Valor de $\mathrm{p}$ \\
\hline \multicolumn{6}{|c|}{ Falta por motivo de saúde no último ano } \\
\hline Não & 41,9 & 79 & 1,00 & - & - \\
\hline $\operatorname{Sim}$ & 58,1 & 69,1 & 0,87 & $0,76-1,00$ & 0,057 \\
\hline \multicolumn{6}{|c|}{ Licença por motivo de saúde no último ano } \\
\hline Não & 54,8 & 76,7 & 1,00 & - & - \\
\hline $\operatorname{Sim}$ & 45,2 & 70,2 & 0,92 & $0,80-1,05$ & 0,218 \\
\hline \multicolumn{6}{|c|}{ Diagnóstico de enfermidade laboral } \\
\hline Não & 86,9 & 73,8 & 1,00 & - & - \\
\hline $\operatorname{Sim}$ & 13,1 & 73,7 & 1,00 & $0,81-1,22$ & 0,987 \\
\hline \multicolumn{6}{|l|}{ Diagnóstico de DCNT } \\
\hline Não & 59 & 74,9 & 1,00 & - & - \\
\hline Sim & 41 & 72,3 & 0,97 & $0,84-1,11$ & 0,626 \\
\hline \multicolumn{6}{|l|}{ Diagnóstico de DIP } \\
\hline Não & 92,8 & 73,2 & 1,00 & - & - \\
\hline Sim & 7,2 & 81 & 1,11 & $0,89-1,38$ & 0,372 \\
\hline \multicolumn{6}{|l|}{ Diagnóstico de DAR } \\
\hline Não & 64,8 & 75,5 & 1,00 & - & - \\
\hline Sim & 35,2 & 70,6 & 0,93 & $0,80-1,09$ & 0,375 \\
\hline \multicolumn{6}{|l|}{ TMC } \\
\hline Não & 72,8 & 81,5 & 1,00 & - & - \\
\hline $\operatorname{Sim}$ & 27,2 & 53,2 & 0,65 & $0,52-0,81$ & $<0,001$ \\
\hline \multicolumn{6}{|l|}{ Diagnóstico de LER/DORT } \\
\hline Não & 91 & 73,5 & 1,00 & - & - \\
\hline Sim & 9 & 76,9 & 1,05 & $0,84-1,31$ & 0,688 \\
\hline \multicolumn{6}{|l|}{ Diagnóstico de lombalgia } \\
\hline Não & 70,3 & 76 & 1,00 & - & - \\
\hline Sim & 29,7 & 68,6 & 0,90 & $0,77-1,06$ & 0,219 \\
\hline
\end{tabular}

Nota: DCNT = Doenças Crônicas Não Transmissíveis; DIP = Doenças Infecciosas e Parasitárias; DAR = Doenças do Aparelho Respiratório; TMC = Transtornos Mentais Comuns; LER/DORT = Lesão por Esforço Repetitivo/Doenças Osteoarticulares Relacionadas ao Trabalho.

fermeiros, auxiliares e técnicos de enfermagem. A probabilidade de TMC (SRQ-20) diminuiu a satisfação do profissional com o seu trabalho. As associações observadas eram esperadas, pois, ambiente, estrutura e organização dos serviços de saúde produzem impacto direto sobre a satisfação do pessoal de enfermagem ${ }^{12,18}$. Esses resultados são relevantes, pois, encontra-se suficientemente estabilizado o conhecimento sobre os prováveis efeitos das associações desse tipo sobre qualidade dos serviços prestados ${ }^{27,28}$.

A porcentagem de satisfeitos foi maior no estrato dos auxiliares e técnicos $(76,2 \%)$ quando comparada ao estrato dos enfermeiros $(71,4 \%)$. Tal resultado é convergente. Quando estudados os componentes da satisfação, os autores identificaram que eles variam de acordo com a posição/ cargo ocupado ${ }^{29}$. Essa associação possivelmente seja eco do grau de empoderamento dos profissionais para agir nas situações de trabalho, por exemplo. Mas, satisfação tem a ver com o nível de escolaridade, que por sua vez, associa-se ao tipo de cargo ocupado. Veja-se que o estrato dos auxiliares é caracterizado por menor escolaridade se comparado ao estrato dos enfermeiros. Diante da disputa no mercado de trabalho brasileiro, com menos chance de emprego para os menos escolarizados $^{30}$, provavelmente, os auxiliares, em sua maioria, com nível médio de escolaridade, tenham aproximado suas expectativas de estar empregado e ter a oportunidade de exercer uma profissão ao vínculo profissional atual. Sabe-se que a satisfação em qualquer dimensão da vida depende da distância entre o que se tem e aquilo que se almeja ${ }^{19}$. No caso dos enfermeiros (todos com nível superior de escolaridade), é possível 
Tabela 3. Condições de trabalho e suas relações com a satisfação no trabalho da enfermagem em uma rede municipal de saúde, 2008/2009.

\begin{tabular}{|c|c|c|c|c|c|}
\hline Variáveis & $\begin{array}{c}\text { Amostra } \\
(\%)\end{array}$ & $\begin{array}{c}\text { Satisfeito com } \\
\text { trabalho }(\%)\end{array}$ & $\mathbf{R P}$ & IC 95\% & $\begin{array}{l}\text { Valor } \\
\text { de p }\end{array}$ \\
\hline \multicolumn{6}{|l|}{ Categoria profissional } \\
\hline Enfermeiro & 50,7 & 71,4 & 1,00 & - & - \\
\hline Auxiliar/Técnico de enfermagem & 49,3 & 76,2 & 1,07 & $0,93-1,22$ & 0,354 \\
\hline \multicolumn{6}{|l|}{ Tempo no trabalho atual (meses) } \\
\hline$<12$ & 20 & 70,7 & 1,00 & - & - \\
\hline 12 a 23 & 9 & 80,8 & 1,14 & $0,89-1,47$ & 0,297 \\
\hline 24 a 47 & 12,1 & 60 & 0,85 & $0,62-1,17$ & 0,312 \\
\hline$\geq 48$ & 59 & 76,6 & 1,08 & $0,90-1,30$ & 0,396 \\
\hline \multicolumn{6}{|l|}{ Carga horária semanal (horas) } \\
\hline Até 40 & 60,3 & 77,1 & 1,00 & - & - \\
\hline Mais de 40 & 39,7 & 68,7 & 0,89 & $0,77-1,03$ & 0,124 \\
\hline \multicolumn{6}{|l|}{ Trabalho noturno } \\
\hline Não & 75,5 & 75,8 & 1,00 & - & - \\
\hline Sim & 24,5 & 67,6 & 0,89 & $0,75-1,07$ & 0,207 \\
\hline \multicolumn{6}{|l|}{ Demanda-controle do trabalho } \\
\hline Baixa exigência & 19,3 & 82,1 & 1,00 & - & - \\
\hline Ativo & 24,1 & 65,7 & 0,80 & $0,65-0,99$ & 0,036 \\
\hline Passivo & 33,8 & 79,6 & 0,97 & $0,83-1,14$ & 0,696 \\
\hline Alta exigência & 22,8 & 66,7 & 0,81 & $0,66-1,00$ & 0,052 \\
\hline \multicolumn{6}{|l|}{ Apoio social no trabalho } \\
\hline Baixo & 49,7 & 66 & 1,00 & - & - \\
\hline Alto & 50,3 & 81,5 & 1,24 & $1,07-1,42$ & 0,003 \\
\hline \multicolumn{6}{|c|}{ Relação exigência das tarefas/recursos disponíveis } \\
\hline Boa & 30 & 83,9 & 1,00 & - & - \\
\hline Regular & 56,6 & 72,6 & 0,86 & $0,76-0,99$ & 0,031 \\
\hline Ruim/Muito ruim & 13,5 & 56,4 & 0,67 & $0,50-0,90$ & 0,008 \\
\hline \multicolumn{6}{|l|}{ Condições do ambiente de trabalho } \\
\hline Precárias & 33,5 & 63,9 & 1,00 & - & - \\
\hline Razoáveis & 40,3 & 76,1 & 1,19 & $0,99-1,43$ & 0,060 \\
\hline Ótimas & 26,2 & 82,9 & 1,30 & $1,08-1,55$ & 0,005 \\
\hline \multicolumn{6}{|l|}{ Carga física } \\
\hline Baixa & 35,5 & 79,6 & 1,00 & - & - \\
\hline Média & 39,3 & 75,4 & 0,95 & $0,82-1,09$ & 0,462 \\
\hline Alta & 25,2 & 63 & 0,79 & $0,65-0,97$ & 0,023 \\
\hline \multicolumn{6}{|l|}{ Demanda emocional } \\
\hline Baixa & 15,2 & 81,8 & 1,00 & - & - \\
\hline Alta & 84,8 & 72,4 & 0,88 & $0,75-1,04$ & 0,131 \\
\hline \multicolumn{6}{|c|}{ Exposição a agentes biológicos/químicos } \\
\hline Baixa & 33,5 & 74,2 & 1,00 & - & - \\
\hline Média & 36,6 & 76,4 & 1,03 & $0,88-1,21$ & 0,719 \\
\hline Alta & 30 & 70,1 & 0,94 & $0,79-1,13$ & 0,537 \\
\hline \multicolumn{6}{|c|}{ Comprometimento excessivo com o trabalho } \\
\hline Baixo & 43,8 & 81,9 & 1,00 & - & - \\
\hline Médio & 24,5 & 78,9 & 0,96 & $0,83-1,11$ & 0,614 \\
\hline Alto & 31,7 & 58,7 & 0,72 & $0,59-0,87$ & 0,001 \\
\hline
\end{tabular}


Tabela 4. Modelo final de regressão múltipla de Poisson com variâncias robustas de variáveis associadas à satisfação no trabalho da enfermagem numa rede municipal de saúde, 2008/2009.

\begin{tabular}{llcc}
\hline \multicolumn{1}{c}{ Variáveis } & RP & IC 95\% & Valor de p \\
\hline Idade (anos) ${ }^{* \dagger}$ & 1,01 & $1,00-1,02$ & 0,002 \\
Apoio social no trabalho & & & - \\
$\quad$ Baixo & 1,00 & - & 0,020 \\
Alto & 1,17 & $1,03-1,34$ & - \\
Condições do ambiente de trabalho ${ }^{\ddagger}$ & & & 0,049 \\
$\quad$ Precárias & 1,00 & - & 0,013 \\
Satisfatórias & 1,18 & $1,00-1,39$ & - \\
Ótimas & 1,25 & $1,05-1,47$ & $<0,001$ \\
TMC & & & - \\
Não & 1,00 & $0,55-0,84$ & \\
Sim & 0,68 & & \\
\hline
\end{tabular}

Nota: ${ }^{*}$ Idade inserida como variável contínua; ${ }^{\circ}$ Limite inferior do IC 95\% = 1,004; ${ }^{*}$ Limite inferior do IC 95\% = 1,0007.

que suas expectativas sejam mais ambiciosas com reflexos sobre o julgamento menos favorável da situação laboral em que se encontram. Não foram, contudo, colhidos insumos para aprofundar essas hipóteses.

Além da escolaridade, antiguidade no emprego e idade têm relação com a satisfação no trabalho. Sabe-se que a experiência traz efeitos positivos, tanto no que concerne à percepção de segurança, quanto às habilidades para desenvolver as tarefas com resultados favoráveis nos fazimentos laborais $^{29}$. Se for assim, os mais velhos estariam mais satisfeitos porque alcançaram equilíbrio entre as demandas e os modos operatórios graças aos atributos que a experiência lhes conferiu? Vale a pena pesquisa futura nesse quesito.

Renda e benefícios monetários são fatores comumente associados à satisfação no trabalho ${ }^{2}$. Mas, surpreendentemente, esse resultado não foi observado. Tal disparidade pode ser em função das opções metodológicas no desenho da pesquisa. Distinto dos estudos que indagam sobre o salário do respondente, optou-se por perguntar sobre a renda familiar. Essa estratégia buscou contornar problemas relacionados à homogeneidade da amostra, no tocante ao aporte salarial e aos demais atributos do contrato de trabalho. Provavelmente, a estratégia provocou conteúdos latentes que explicariam a falta de associação entre renda familiar e satisfação no trabalho.

Saúde mental e satisfação são dois construtos muito próximos um do outro, sendo influenciadas pelas condições de trabalho ${ }^{31}$. Dessa forma, a associação negativa entre satisfação no trabalho e TMC era esperada. Vale mencionar que tais transtornos dizem respeito a um conjunto de sintomas (queixas somáticas, fadiga, esquecimento, insônia, irritabilidade e falta de concentração), que por si só não constituem um quadro nosológico, mas que representam um estado psíquico menos saudável acompanhado de sentimentos negativos, como é o caso da insatisfação no trabalho $^{32,33}$.

Os resultados descritos justificam a importância de problematizar o modelo e a estrutura da organização dos serviços, com vistas a identificar bloqueios à atividade do pessoal de enfermagem. Pesquisas sugerem apoio da gestão com objetivo de melhorar a comunicação entre os diferentes profissionais e promover um ambiente "amistoso", em vez de criar mecanismos que geram controle burocrático sobre os fazimentos laborais ${ }^{5}$. Além disso, tentar adequar o ambiente físico e equipamentos às necessidades do trabalho e dos trabalhadores, ou, ao menos reforçar o interesse em identificar falhas nesse âmbito, foi anteriormente sugerido ${ }^{2}$. Avaliação das margens existentes para os trabalhadores se envolverem nos assuntos relacionados ao serviço, existência de medidas para promoção da qualidade da assistência, esforços para a adequação dos recursos e infraestrutura, e, por fim, qualidade do relacionamento com os médicos, entre outros, são medidas exequíveis ${ }^{20}$. Essas estratégias provocam impacto positivo sobre a satisfação no trabalho, na medida em que favorecem a percepção de apoio ao desenvolvimento das tarefas ${ }^{34}$.

As condições que o pessoal de enfermagem encontra para responder às demandas e mobilizar as suas competências podem estar associa- 
das aos resultados alcançados. A falta de apoio para desenvolver as tarefas, ao seu turno, pode contrariar a gestão dos elementos que os enfermeiros tentam estruturar'. Na divisão técnica do trabalho em saúde, esses profissionais assumem o gerenciamento do cuidado em um sistema técnico-organizacional bastante complexo. Vale lembrar que, constantemente, os enfermeiros são chamados para resolver problemas de toda ordem. Sendo assim, são requeridos conhecimentos variados, competências em um largo campo de atuação, responsabilidade e capacidade de decisão no processo de ordenação das ativida$\mathrm{des}^{35}$. De que maneira eles podem ser apoiados? Resultados anteriores identificaram a relevância do apoio institucional, por exemplo, formação centrada nas dimensões da prática. No Brasil, a experiência do Curso de Gestão das Condições de Trabalho e Saúde dos Trabalhadores da Saúde (Cegest) obteve resultados consistentes ${ }^{36}$. Foi sugerido aos gestores enfermeiros ampliar a escuta e buscar meios para melhorar as relações dos auxiliares e dos técnicos de enfermagem com os médicos, por exemplo ${ }^{12,37}$.

Quanto à transformação das condições de trabalho, está indicada a formação de comitês para desenvolver intervenções participativas nos ambientes laborais de maneira a estabelecer prioridades em consonância com as necessidades diferenciadas das missões em curso. As diretrizes da Política Nacional de Promoção da Saúde do Trabalhador do SUS ${ }^{38}$ incluem o incentivo à formação dos trabalhadores para agir nos comitês locais visando a melhoria dos ambientes.

Seria prudente avaliar de forma sistemática os fatores ambientais como ventilação, temperatura, iluminação, ruído ${ }^{37}$. Quanto aos recursos técnicos e equipamentos, mais uma vez, indica-se a estratégia de estimular a participação dos próprios trabalhadores ${ }^{12,39}$ para avaliar a disponibilidade e a qualidade dos recursos e equipamentos que eles encontram para trabalhar. Por fim, se os serviços de promoção da saúde e vigilância dos ambientes de trabalho já foram implantados, programas e intervenções integrados e participativos continuam dependentes das margens que os gestores enfermeiros dispõem para fortalecer essas e outras iniciativas ${ }^{39}$.

A interpretação dos resultados apresentados acima pode ser ambígua porque o estudo seccional não indica a direção das associações en- contradas. Por exemplo, o respondente satisfeito com a sua vida familiar tenderia a julgar de forma mais positiva os resultados do seu trabalho ou as condições que encontra para operar, ambos os critérios são estreitamente relacionados com a percepção de satisfação no trabalho. Ademais, vale também destacar que não foram abordadas situação da vida familiar nem características da personalidade. Essas dimensões influenciam os processos de percepção e avaliação dos contextos pelo sujeito, incluindo a sua percepção sobre o trabalho.

A força do presente estudo foi abordar a percepção dos trabalhadores incluídos em uma amostra aleatória e estratificada de acordo com os diferentes níveis da rede e região geográfica dos serviços por meio de um instrumento que incluiu questões sociodemográficas e ocupacionais. Tal estratégia foi orientada pelos conhecimentos recentes sobre o caráter multidimensional da satisfação laboral. A pesquisa permitiu, pois, avaliar a percepção de satisfação em uma amostra heterogênea quanto às características individuais, cargo, tipo de serviço, local e ambiente laboral. O viés de seleção foi minimizado porque, além da estratégia do sorteio, a taxa de resposta foi alta. A robustez dos resultados foi confirmada, pois as duas técnicas multivariadas de análise de dados produziram resultados semelhantes. A confiabilidade da medida de satisfação examinada por uma única pergunta, no caso "Você está satisfeito com o seu trabalho?", em vez de utilizar escalas validadas já foi autorizada. A metanálise ${ }^{40}$ não identificou diferenças significativas nas estimativas de satisfação comparando resultados de estudos que utilizaram escalas com aqueles que utilizaram uma única pergunta para avaliar essa percepção. Segundo o pesquisador, as medidas de satisfação global com o trabalho aferidas por um único item, provavelmente, são mais robustas do que aquelas aferidas por escalas, porque seriam menos influenciadas por fatores não controláveis.

\section{Conclusão}

A satisfação dos participantes com o trabalho indica a pertinência para a gestão de enfocar as condições ambientais e psicossociais de trabalho (apoio social, no caso) com prováveis vantagens para o bem-estar do pessoal da enfermagem. 


\section{Colaboradores}

AA Assunção coordenou o estudo e elaborou a estrutura do artigo. AM Pimenta analisou os dados. Ambos autores participaram da redação, revisão crítica e aprovação da versão final do artigo.

\section{Agradecimentos}

À FAPEMIG (Fundação de Amparo a Pesquisa de Minas Gerais) e ao CNPq (Conselho Nacional de Desenvolvimento Científico e Tecnológico).

\section{Referências}

1. Shin JI, Lee E. The effect of social capital on job satisfaction and quality of care among hospital nurses in South Korea. J Nurs Manag 2016; 24(7):934-942.

2. Twigg D, McCullough K. Nurse retention: A review of strategies to create and enhance positive practice environments in clinical settings. Inter J Nurs Stud 2014; 51(1):85-92.

3. Rizzotto MLF, Gil CRR, Carvalho MD, Fonseca ALN, Santos MF. Força de trabalho e gestão do trabalho em saúde: revelações da Avaliação Externa do Programa Nacional de Melhoria do Acesso e da Qualidade da Atenção Básica no Paraná. Saude Debate 2014; 38:237251.

4. Portela GZ, Fehn AC, Ungerer RLS, Poz MRD. Human resources for health: global crisis and international cooperation. Cien Saude Colet 2017; 22(7):2237-2246.

5. Li H, Shi Y, Li Y, Xing Z, Wang S, Ying J, Zhang M, Sun J. Relationship between nurse psychological empowerment and job satisfaction: A systematic review and meta analysis. J Adv Nurs 2018; 74(6):1264-1277.

6. Magalhães AMM, Dall'Agnol CM, Marck PB. Nursing workload and patient safety - a mixed method study with an ecological restorative approach. Rev Lat Am Enfermagem 2013; 21(n. esp.):146-154.

7. Carvalho MD, Santos NRD, Campos GWDS. A construção do SUS e o planejamento da força de trabalho em saúde no Brasil: breve trajetória histórica. Saude Debate 2013; 37:372-387.

8. Khademi M, Mohammadi E, Vanaki Z. Resourcestasks imbalance: Experiences of nurses from factors influencing workload to increase. Iran J Nurs Midwifery Res 2015; 20(4):476-483.

9. Assunção AA, Jackson Filho JM. Transformações do trabalho no setor saúde e condições para cuidar. In: Assunção AA, Brito JC, organizadoras. Trabalhar na Saúde: experiências cotidianas e desafios para a gestão do trabalho e do emprego. Rio de Janeiro: Fiocruz; 2011. p. 45-65.

10. Boafo IM. The effects of workplace respect and violence on nurses' job satisfaction in Ghana: a cross-sectional survey. $H R H$ 2018; 16(1):6.

11. Sarafis P, Rousaki E, Tsounis A, Malliarou M, Lahana L, Bamidis P, Niakas D, Papastavrou E. The impact of occupational stress on nurses' caring behaviors and their health related quality of life. BMC Nurs 2016; 15:56.

12. Nantsupawat A, Kunaviktikul W, Nantsupawat R, Wichaikhum OA, Thienthong H, Poghosyan L. Effects of nurse work environment on job dissatisfaction, burnout, intention to leave. Inter Nurs Rev 2017; 64(1):91-98.

13. Manyazewal T, Matlakala MC. Beyond patient care: the impact of healthcare reform on job satisfaction in the Ethiopian public healthcare sector. $H R H$ 2017; 15(1):10.

14. Fernet C, Trepanier S-G, Demers M, Austin S. Motivational pathways of occupational and organizational turnover intention among newly registered nurses in Canada. Nurs Outlook 2017; 65(4):444-454.

15. Carvalho MN, Gil CRR, Costa EMOD, Sakai MH, Leite SN. Necessidade e dinâmica da força de trabalho na Atenção Básica de Saúde no Brasil. Cien Saude Colet 2018; 23(1):295-302. 
16. Spector PE. Job Satisfaction. Sage Publications: Thousand Oaks; 1997.

17. Smith JG. The nurse work environment: Current and future challenges. J Appl Biobehav Res 2018; 23(1):e12126.

18. Lu H, Barriball KL, Zhang X, While AE. Job satisfaction among hospital nurses revisited: a systematic review. Int J Nurs Stud 2012; 49(8):1017-1038.

19. Hayes B, Bonner ANN, Pryor J. Factors contributing to nurse job satisfaction in the acute hospital setting: a review of recent literature. $J$ Adv Nurs 2010; 18(7):804-814.

20. Lake ET. Development of the practice environment scale of the Nursing Work Index. Res Nurs Health 2002; 25(3):176-188.

21. Paz Filho GJ, Sato LJ, Tuleski MJ, Takata SY, Ranzi CC, Saruhashi SY, Spadoni B. Use of the CAGE questionnaire for detecting alcohol use disorders in the emergency room. Rev Assoc Med Bras 2001; 47(1):65-69

22. Santos KOB, Araújo TM, Oliveira NF. Factor structure and internal consistency of the Self-Reporting Questionnaire (SRQ-20) in an urban population. Cad Saude Publica 2009; 25:214-222.

23. Barbosa REC, Assunção AA, Araujo TM. Musculoskeletal pain among healthcare workers: An exploratory study on gender differences. Am J Ind Med 2013; 56(10):1201-1212.

24. Karasek R, Brisson C, Kawakami N, Houtman I, Bongers P, Amick B. The Job Content Questionnaire (JCQ): an instrument for internationally comparative assessments of psychosocial job characteristics. $J \mathrm{Oc}$ cup Health Psycho 1998; 3(4):322-355.

25. Alves MG, Chor D, Faerstein E, Lopes CS, Werneck GL. Short version of the "job stress scale": a Portuguese-language adaptation. Rev Saude Publica 2004; 38(2):164-171.

26. Silva LS, Barreto SM. Adaptação transcultural para o português brasileiro da escala effort-reward imbalance: um estudo com trabalhadores de banco. Rev Panam Salud Publica 2010; 27(1):32-36.

27. Kaddourah BT, Khalidi A, Abu-Shaheen AK, Al-Tannir MA. Factors impacting job satisfaction among nurses from a tertiary care centre. J Clin Nurs 2013; 22(21-22):3153-3159.

28. Ishara S, Bandeira M, Zuardi AW. Public psychiatric services: job satisfaction evaluation. Rev Bras Psiquiatria $2008 ; 30(1): 38-41$.

29. Cheung K, Ching SSY. Job satisfaction among nursing personnel in Hong Kong: a questionnaire survey. $J$ Adv Nurs 2014; 22(5):664-675.

30. Santos ALD, Gimenez D. Inserção dos jovens no mercado de trabalho. Estud Av 2015; 29(85):153-168.
31. Arends I, Bültmann U, Nielsen K, van Rhenen W, Boer MR, van der Klink JJL. Process evaluation of a problem solving intervention to prevent recurrent sickness absence in workers with common mental disorders. Soc Sci Med 2014; 100:123-132.

32. Stansfeld SA, Fuhrer R, Head J. Impact of common mental disorders on sickness absence in an occupational cohort study. Occup Environ Med 2011; 68(6):408-413.

33. Rai D, Skapinakis P, Wiles NN, Lewis G, Araya R. Common mental disorders, subthreshold symptoms and disability: longitudinal study. $B r J$ Psychiatry 2010; 197(5):411-412.

34. Lobburi P. The influence of organizational and social support on turnover intention in collectivist contexts. JABR 2011; 28(1):93-104.

35. Marques GQ, Silva-Lima MAD. Organização tecnológica do trabalho em um pronto atendimento e a autonomia do trabalhador de enfermagem. Rev Esc Enf USP 2008; 42(1):41-47.

36. Assunção AA, Pimenta AM, Harayama RM. Relatório técnico: barreiras e facilitadores na implementação da política nacional de promoção da saúde dos trabalhadores do SUS. Belo Horizonte: UFMG; 2017.

37. Yang Y, Liu YH, Liu JY, Zhang HF. The impact of work support and organizational career growth on nurse turnover intention in China. Int J Nurs Scien 2015; 2(2):134-139.

38. Brasil. Protocolo no 008, de 01 de dezembro de 2011. Mesa Nacional de Negociação Permanente do SUS. Institui as diretrizes da Política Nacional de Promoção da Saúde do Trabalhador do Sistema Único de SaúdeSUS. Brasília: MS; 2011.

39. Henning R, Warren N, Robertson M, Faghri P, Cherniack M, CPH-NEW Research Team. Workplace health protection and promotion through participatory ergonomics: an integrated approach. Public Health Rep 2009; 124(Supl. 1):26-35.

40. Wanous JP, Reichers AE, Hudy MJ. Overall job satisfaction: how good are single-item measures? J Appl Psycho 1997; 82(2):247-252.

Artigo apresentado em 07/04/2019

Aprovado em 20/08/2019

Versão final apresentada em 30/09/2019 